\title{
Author Correction: Areas of global importance for conserving terrestrial biodiversity, carbon and water
}

Martin Jung (1), Andy Arnell(1), Xavier de Lamo, Shaenandhoa García-Rangel, Matthew Lewis (i), Jennifer Mark (1), Cory Merow, Lera Miles (D), lan Ondo (D), Samuel Pironon (1), Corinna Ravilious, Malin Rivers (D), Dmitry Schepaschenko (D), Oliver Tallowin, Arnout van Soesbergen, Rafaël Govaerts (D), Bradley L. Boyle, Brian J. Enquist (D), Xiao Feng (D),

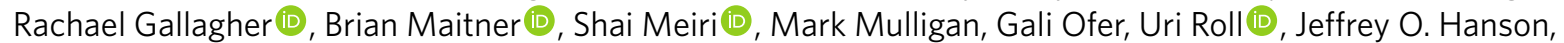
Walter Jetz (1), Moreno Di Marco, Jennifer McGowan (D), D. Scott Rinnan (D, Jeffrey D. Sachs, Myroslava Lesiv (1),

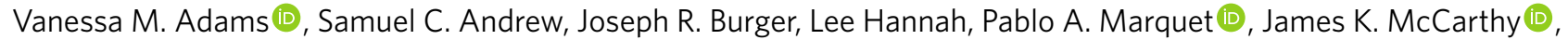
Naia Morueta-Holme (i), Erica A. Newman, Daniel S. Park (D), Patrick R. Roehrdanz (1), Jens-Christian Svenning (iD,

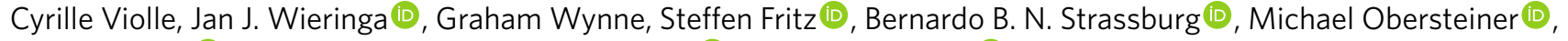
Valerie Kapos (D), Neil Burgess, Guido Schmidt-Traub (i) and Piero Visconti(D)

Correction to: Nature Ecology \& Evolution https://doi.org/10.1038/s41559-021-01528-7, published online 23 August 2021.

In the version of this Article initially published, an error appeared in the spelling of the thirteenth author's name. Specifically, author Dmitry Schepaschenko's name initially appeared as "Dmitry Schepashenko." This has now been corrected in the online version of the Article.

Published online: 23 September 2021

https://doi.org/10.1038/s41559-021-01569-y

(c) The Author(s), under exclusive licence to Springer Nature Limited 2021 